\title{
Accuracy of Serum Golgi Protein 73 and Alpha Fetoprotein (AFP) to Diagnose Hepatocellular Carcinoma
}

\author{
Karina Dwi Swastika*, Gontar Alamsyah Siregar**, Dharma Lindarto*** \\ *Resident in Department of Internal Medicine, Faculty of Medicine, Universitas Sumatera Utara, Medan, \\ Indonesia \\ **Professor in Division of Gastroentero-Hepatology, Department of Internal Medicine, Universitas Sumatera \\ Utara, Haji Adam Malik General Hospital, Medan, Indonesia \\ ****Professor in Division of Endocrine and Metabolic Disease, Department of Internal Medicine, Universitas \\ Sumatera Utara, Haji Adam Malik General Hospital, Medan, Indonesia
}

Corresponding Author: Gontar Alamsyah Siregar

\begin{abstract}
Background: Hepatocellular Carcinoma (HCC) is one of the most common malignancies in the liver. Modalities of diagnostic are often an obstacle in HCC surveillance. Alpha fetoprotein (AFP) is one of protein that often used in the diagnostic of HCC in chronic liver disease. Golgi protein 73 (GP73), is one of the candidate biomarkers in early diagnostic of HCC and found in biliary epithelial cells but rarely expressed by normal hepatocytes. Expression of GP73 was reported to be increased in a large number of malignancies. Aims of this study to evaluate differences in Golgi protein 73 serum (sGP73) and AFP in diagnosing hepatocellular carcinoma in patients with liver cirrhosis.
\end{abstract}

Materials and Methods: This cross-sectional study was conducted at Haji Adam Malik Hospital in 2020. Serum level of GP73 and others biomarker was detected using enzymelike immunosorbent assay.

Results: From 90 subjects, Liver cirrhosis and HCC group had significantly higher AFP than the control group. AFP was superior in determining $\mathrm{HCC}$ to GP73. At a cut off value of $>394.5 .00 \mathrm{ng} / \mathrm{mL}$, AFP yielded a sensitivity of $83.3 \%$ and specificity of $67 \%$, for discriminating liver cirrhosis and HCC (AUC 0.84 ), while GP73 with cut off value of $>82.5$ $\mathrm{ng} / \mathrm{mL}$, sensitivity of $70 \%$ and specificity of 57\% (AUC 0.74).

Conclusion: GP73 was significantly higher in $\mathrm{HCC}$ patients in comparison to non-HCC patients and healthy population. Compared with alpha fetoprotein, GP73 was superior in discriminating $\mathrm{HCC}$ in healthy population but inferior in group of liver cirrhosis.

Keywords: Golgi Protein 73, Alpha Fetoprotein, Hepatocellular carcinoma

\section{INTRODUCTION}

Hepatocellular Carcinoma (HCC) is one of the most common primary malignant in the liver and placed in the third position of the most common mortality in malignancy. Usually, HCC is caused by chronic liver diseases such as chronic hepatitis and liver cirrhosis. In dealing with a tumor such as HCC, finding it as early as possible is an ideal step. ${ }^{1}$

In developing countries like Indonesia, modalities of diagnostic are often an obstacle in HCC surveillance. Alpha fetoprotein (AFP) is one of protein produced by developing baby and often used in supporting the diagnostic of $\mathrm{HCC}$ in chronic liver disease. However, AFP is found to be normal in $40 \%$ of HCC patients, especially at an early stage, so this examination is considered to have a fairly low sensitivity and specificity. ${ }^{2}$

Golgi protein 73 (GP73), is one of the candidate biomarkers that has been widely studied for its usefulness in early 
diagnostic of HCC. GP73 is a type II Golgi membrane protein that is commonly found in biliary epithelial cells but very rarely expressed by normal hepatocytes. Expression of GP73 was reported to be increased in a large number of malignancies, such as seminoma, renal cell carcinoma, and lung adenocarcinoma. The expression of GP73 is reported to have a very close relationship with HCC. Even though pathogenesis of liver carcinogenesis is still unclear, but the upregulating of this protein is found underlying liver disease due to viral hepatitis infection, alcohol-induced liver disease and cirrhosis. Iftikhar et al., (2004) revealed that the increasing of GP73 expression originates from activated stellate cells. ${ }^{3}$ The aims of this study to evaluate differences in Golgi protein 73 serum (sGP73) and AFP in all patients with liver cirrhosis with hepatocellular carcinoma and without hepatocellular carcinoma and evaluate the diagnostic accuracy of sGP73 in diagnosing hepatocellular carcinoma in patients with liver cirrhosis.

\section{METHODS}

This is a cross sectional study conducted at Haji Adam Malik Hospital and other satellite Hospital. The protocol was approved by Health Research Ethics Commission of Medical Faculty of University of Sumatera Utara and Haji Adam Malik Hospital Medan. Study was carried out in 2020.

\section{Patient selection}

The Inclusion criteria included: Men and women who were not pregnant aged > 18 years and diagnosed with cirrhosis from the results of ultrasound examination and willing to participate in the study. All patients with systemic disease and other malignancies causing elevation of AFP levels were excluded from the study. All patients undergo some hematologic and biochemical tests related to liver function, HCV antibodies, HBsAg, AFP, and radiological examinations in the form of ultrasound and triple phase CT of the liver.
Examination of GP73 was assessed using the ELISA kit.

\section{Definition of cirrhosis and hepatocellular carcinoma}

Patients were defined as cirrhosis based on the finding of nodular liver surface, round edge, and hypoechoic nodules of liver parenchyma in ultrasound. Hepatocellular carcinoma based on the finding of hypervascularity in the arterial phase, washout in the portal vein phase or delayed phase in triple phase CT of the liver.

\section{Statistical methods}

The characteristics of each variable were presented in the central measure (mean) and distribution variation (standard deviation or range). The normality of data was assessed using the Shapiro-Wilk test. Numerical data were described as Mean \pm SD if normally distributed or median and interquartile range (IQR) if not normally distributed. Sensitivity and specificity of data were analyzed by using a Receiver Operating Curve in SPSS $22^{\text {nd }}$ version (SPSS Inc., Chicago).

\section{RESULTS}

\section{Clinical characteristics of the patient}

Of the 90 patients (30 patients with liver cirrhosis, 30 patients with HCC and 30 control group) who met the inclusion and exclusion criteria, mean of age was $53,7 \pm 21.65$ years in liver cirrhosis, $52,97 \pm 18.40$ in HCC and $32,43 \pm 29.12$ in control group. The most etiology of $\mathrm{SH}$ and HCC was Hepatitis B virus (56.6\% in SH and $73.3 \%$ in HCC). Laboratory data showed means of AFP were $485 \pm 621.65$ $\mathrm{ng} / \mathrm{ml}$ in $\mathrm{SH}$ group, $191886 \pm 807619.68$ in HCC group and $163.03 \pm 12.09$ in control group with $p$ value $<0.001$ and mean of GP 73 were $75.63 \pm 17.27 \mathrm{ng} / \mathrm{ml}$ in $\mathrm{SH}$ group, $100.53 \pm 30.83$ in HCC group and in 39.37 \pm 11.929 control group with $\mathrm{p}$ value $<0.001$. Liver cirrhosis patients and hepatocellular carcinoma patients had a significantly higher AFP and GP73 than control group. 
Karina Dwi Swastika et.al. Accuracy of serum golgi protein 73 and alpha fetoprotein (AFP) to diagnose hepatocellular carcinoma.

\begin{tabular}{l} 
Table. 1 Clinical sharacteristics of the patients \\
\begin{tabular}{|l|l|l|l|l|}
\hline & SH $(\mathbf{n}=\mathbf{3 0})$ & HCC $(\mathbf{n}=\mathbf{3 0})$ & Control $(\mathbf{n}=\mathbf{3 0})$ & P value \\
\hline Age & $53,7 \pm 21.65$ & $52,97 \pm 18.40$ & $32,43 \pm 29.12$ & \\
\hline Etiology (n (\%)) & & & & \\
\hline Hep B & $17(56.6)$ & $22(73.3)$ & & \\
\hline Hep C & $2(6.6)$ & $7(23.3)$ & & \\
\hline Alcoholic & $11(36.6)$ & $1(3.3)$ & & \\
\hline AFP (Mean \pm SD) & $485 \pm 621.65$ & $191886 \pm 807619.68$ & $163.03 \pm 12.09$ & $<0.001$ \\
\hline GP73 (Mean \pm SD) & $75.63 \pm 17.27$ & $100.53 \pm 30.83$ & $39.37 \pm 11.929$ & $<0.001$ \\
\hline
\end{tabular} \\
\\
a Numerical data, normal distribution : mean \pm SD \\
${ }^{b}$ Numerical data, abnormal distribution : median (min-max) \\
\hline
\end{tabular}

\section{Diagnostic accuracy of AFP and GP73}

Alpha fetoprotein (AFP) was a significant discriminator of HCC. At a cut off value of $>200.50 \mathrm{ng} / \mathrm{mL}$, AFP yielded a sensitivity of $96.7 \%$ and specificity of $73.3 \%$ for the discrimination between HCC and normal populations. Similarly at a cut off value of $>53 \mathrm{ng} / \mathrm{mL}$, GP 73 yielded a sensitivity of $96.7 \%$ and specificity of $86.7 \%$ for the discrimination between HCC and normal populations. Comparison of ROC curved between AFP and GP 73 as a discriminator of HCC showed area under curved (AUC) of GP 73 was 0.98 and area under curved (AUC) of AFP was 0.95. (Figure 1 and Table 2)

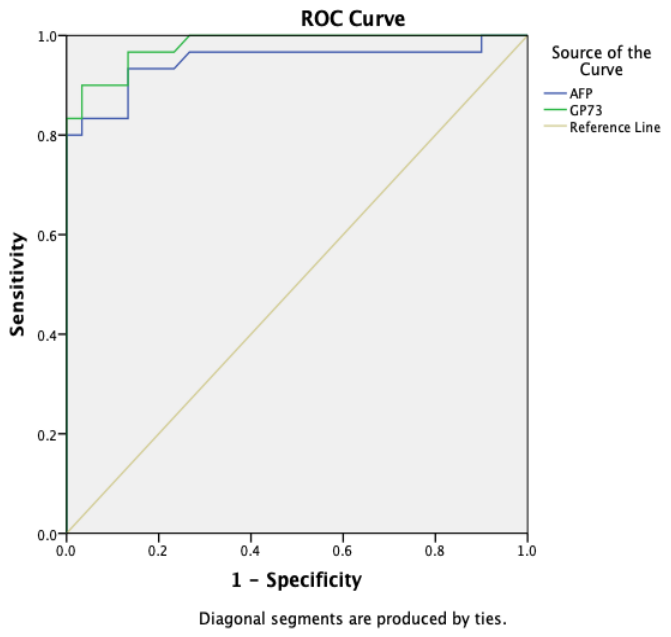

Figure 1. ROC curved of AFP and GP 73 between HCC and normal population

Table 2. The Area Under the Curved and Diagnostic Accuracy of AFP and GP 73 in HCC versus normal population

\begin{tabular}{|l|l|l|l|l|l|}
\hline Variable & AUC & P-value & Cut-off points & Sensitivity & Specificity \\
\hline AFP & 0.95 & $<0.001$ & $\geq 200.50 \mathrm{ng} / \mathrm{ml}$ & $96.7 \%$ & $73.3 \%$ \\
\hline GP 73 & 0.98 & $<0.001$ & $\geq 53 \mathrm{ng} / \mathrm{ml}$ & $96.7 \%$ & $86.7 \%$ \\
\hline
\end{tabular}

Alpha fetoprotein (AFP) was a significant discriminator of liver cirrhosis. At a cut off value of $>158.00 \mathrm{ng} / \mathrm{mL}$, AFP yielded a sensitivity of $90.0 \%$ and specificity of $57 \%$ for the discrimination between liver cirrhosis and normal populations. Similarly, at a cut off value of $>51.5 \mathrm{ng} / \mathrm{mL}$, GP 73 yielded a sensitivity of $90 \%$ and specificity of $87 \%$ for the discrimination between liver cirrhosis and normal populations. Comparison of ROC curved between AFP and GP 73 as a discriminator of liver cirrhosis showed area under curved (AUC) of GP 73 was 0.95 and area under curved (AUC) of AFP was 0.79. (Figure 2 and table 3 )

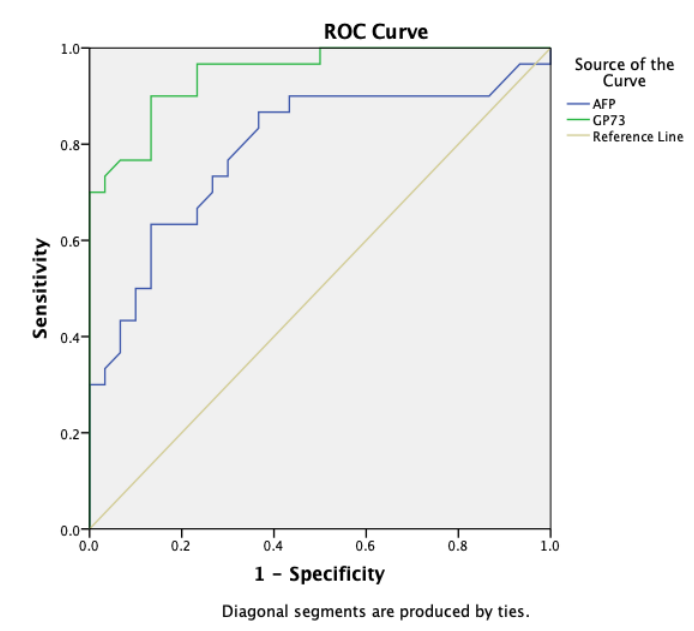

Figure 2. ROC curved of AFP and GP 73 between liver cirrhosis and normal population

Table 3 The Area Under the Curved and Diagnostic Accuracy of AFP and GP 73 in Liver Cirrhosis versus normal population

\begin{tabular}{|l|l|l|l|l|l|}
\hline Variable & AUC & P-value & Cut-off points & Sensitivity & Specificity \\
\hline AFP & 0.79 & $<0.001$ & $\geq 158.00 \mathrm{ng} / \mathrm{ml}$ & $90.0 \%$ & $57 \%$ \\
\hline GP 73 & 0.94 & $<0.001$ & $\geq 51.5 \mathrm{ng} / \mathrm{ml}$ & $90 \%$ & $87 \%$ \\
\hline
\end{tabular}


Alpha fetoprotein (AFP) was a significant discriminator of liver cirrhosis and hepatocellular carcinoma. At a cut off value of > 394.5.00 ng/mL, AFP yielded a sensitivity of $83.3 \%$ and specificity of $67 \%$ for the discrimination between liver cirrhosis and hepatocellular carcinoma. Similarly, at a cut off value of $>82.5$ $\mathrm{ng} / \mathrm{mL}$, GP 73 yielded a sensitivity of $70 \%$ and specificity of $57 \%$ for the discrimination between liver cirrhosis and hepatocellular carcinoma. Comparison of ROC curved between AFP and GP 73 as a discriminator of liver cirrhosis and hepatocellular carcinoma showed area under curved (AUC) of GP 73 was 0.74 and area under curved (AUC) of AFP was 0.84

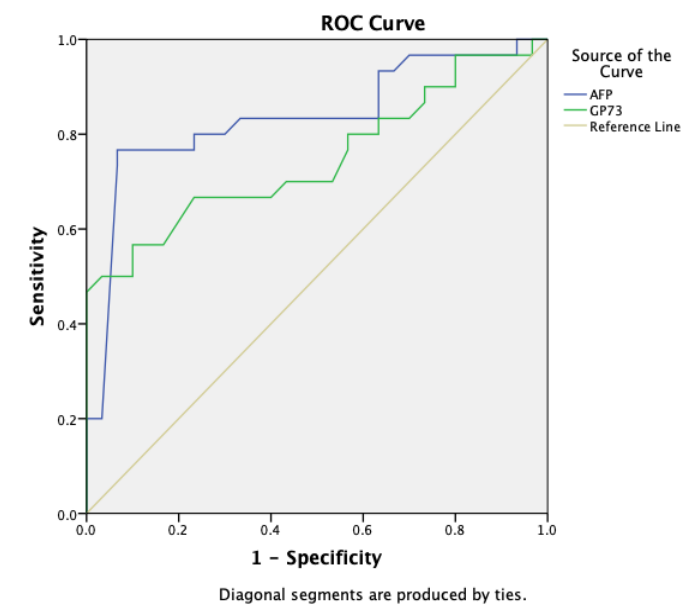

Figure 3. ROC curved of AFP and GP 73 between liver cirrhosis and hepatocellular carcinoma

Table 4 The Area Under the Curved and Diagnostic Accuracy of AFP and GP 73 in Liver Cirrhosis versus Hepatocellular carcinoma

\begin{tabular}{|l|l|l|l|l|l|}
\hline Variable & AUC & P-value & Cut-off points & Sensitivity & Specificity \\
\hline AFP & 0.84 & $<0.001$ & $\geq 394.5 \mathrm{ng} / \mathrm{ml}$ & $83.3 \%$ & $67.0 \%$ \\
\hline GP 73 & 0.73 & $<0.001$ & $\geq 82.5 \mathrm{ng} / \mathrm{ml}$ & $70.0 \%$ & $57.0 \%$ \\
\hline
\end{tabular}

\section{DISCUSSION}

Ninety subjects who were divided in equal ratio into the hepatocellular carcinoma (HCC) group (30), Liver cirrhosis (30) and control group (30). The means age of patients were $52,97 \pm 18.40$ years in $\mathrm{HCC}$ group; $53,7 \pm 21.65$ years in liver cirrhosis; and $32,43 \pm 29.12$ in control group. This result was similar to Liu et al in 2017 which showed older age above 50 years was a certain risk factor for HCC. The most etiology of liver cirrhosis and hepatocellular carcinoma was hepatitis B virus $(56.6 \%$ and $73.3 \%){ }^{4}$ Westwood et al in 2013 showed chronic hepatitis $\mathrm{B}$ and $\mathrm{C}$ were the most common causes of chronic hepatitis in the world. $^{5}$ In line with our finding Mehinovic L et al in 2018 found chronic hepatitis B was the main etiology of $\mathrm{HCC}$ and liver cirrhosis. $^{6}$

In our study, we found that hepatocellular carcinoma and liver cirrhosis patients had a significantly higher level of AFP and GP 73 than normal patient with the highest level of AFP and GP 73 was in the HCC patients (191886 \pm 807619.68 in HCC patients, $485 \pm 621.65$ in liver cirrhosis and $163.03 \pm 12.09$ in normal patients for AFP;
$100.53 \pm 30.83$ in HCC patients, 75.63 \pm 17.27 in liver cirrhosis and $39.37 \pm 11.929$ in normal group for GP73). Recently, Omaima et al in 2020 evaluated that Hepatocellular carcinoma and liver cirrhosis patient had a significantly higher level of AFP than normal patients

In our study, we found that liver cirrhosis and HCC group had significantly higher AFP than the control group. In addition, patients with HCC had significantly higher AFP than liver cirrhosis patients. Diagnostic accuracy of AFP was relatively high. At a cut-off value of $\geq 200.50 \mathrm{ng} / \mathrm{mL}$, AFP yielded a sensitivity of $96.7 \%$ and specificity of $73.3 \%$ for the discrimination between $\mathrm{HCC}$ and normal populations. Similarly, GP 73 yielded a high accuracy with sensitivity of $96.7 \%$ and specificity of $86.7 \%$ at a cut off value of > $53 \mathrm{ng} / \mathrm{mL}$ between HCC and normal populations. In line with our finding Omaima et al.,2020 found at AFP with cut off value $\geq 2.4 \mathrm{ng} / \mathrm{mL}$ in $\mathrm{HCC}$ patients was significantly higher than normal patients with AUC, 95\% CI 0.85 (0.61-100.0) and GP73 with cut off value $\geq 8.4 \mathrm{ng} / \mathrm{mL}$ in HCC patients was significantly higher than 
normal patients with AUC, $96 \%$ CI 0.0 .96 $(0.92-0.99){ }^{7}$

Our findings showed that serum AFP differentiate liver cirrhosis and HCC. At a cut off value of $>394.5 .00 \mathrm{ng} / \mathrm{mL}$, AFP yielded a sensitivity of $83.3 \%$ and specificity of $67 \%$ for the discrimination between liver cirrhosis and hepatocellular carcinoma. Similarly, at a cut off value of $>$ $82.5 \mathrm{ng} / \mathrm{mL}$, GP 73 yielded a sensitivity of $70 \%$ and specificity of $57 \%$ for the discrimination between liver cirrhosis and hepatocellular carcinoma. Comparison of ROC curved between AFP and GP 73 as a discriminator of liver cirrhosis and hepatocellular carcinoma, AFP was showed better than GP 73 with area under curved (AUC) of GP 73 was 0.74 and area under curved (AUC) of AFP was 0.84. In agreement with our findings, Soresi et al., evaluated the best cut-off value for serum AFP to differentiate between liver cirrhosis and HCC. The area under the ROC curve was $0.81 \pm 0.02$; the best determined cut-off value was $20 \mathrm{ng} / \mathrm{mL}$. At this level, sensitivity was $65 \%$ and specificity $89 \%$. More recently, Omaima et al found AFP was superior in determining HCC to GP73 with AUC 0.84 at cut off value > 394.5.00 ng/mL. ${ }^{8}$

\section{CONCLUSION}

Golgi protein 73 is one of good biomarker for detection of HCC. Our study showed that the level of GP 73 was significantly higher in HCC patients in comparison to non-HCC patients and healthy population. Compared with alpha fetoprotein, GP 73 was superior in discriminating $\mathrm{HCC}$ in healthy population but inferior in group of liver cirrhosis. However, some limitations, such as being a small sample-sized and single-centre study have been considered in our study. So, further and more studies with strict designs, large sample sizes multiple collaboration centers are therefore needed.

\section{ACKNOWLEDGEMENT}

The author would like acknowledge that the present research is supported by the Ministry of Research and technology and Higher Education Republic of Indonesia. The support is under the research gran DRPM (Direktorat Riset dan Pengabdian Masyrakat), with contract number: 11/AMD/E1/KP.PTNBH/2020.

\section{Conflict of Interest: None}

\section{Source of Funding: None}

\section{Ethical Approval: Approved}

\section{REFERENCES}

1. Carr BI, Akkiz H, Üsküdar O, et al. HCC with low-and normal-serum alphafetoprotein levels. Clin Pract. 2018;15(1): 453.

2. Marwoto W, Diana S, Roostini ES. Epidemiology of liver cancer in Indonesia. Southeast Asian J Trop Med Public Health. 1985 Dec;16(4):607-8. PMID: 3012789.

3. Iftikhar R, Kladney RD, Havlioglu N, Schmitt-Graff A, Gusmirovic I, Solomon H, Luxon BA, Bacon BR, Fimmel CJ: Disease and cell-specific expression of gp73 in human liver disease. Am J Gastroenterol 2004;99:1087-1095

4. Liu P, Xie S-H, Hu S, et al. Age-specific sex difference in the incidence of hepatocellular carcinoma in the United States. Oncotarget. 2017;8(40):68131.

5. Westwood M, Joore M, Grutters J, et al. Contrast-enhanced ultrasound using SonoVue®(sulphur hexafluoride microbubbles) compared with contrastenhanced computed tomography and contrast-enhanced magnetic resonance imaging for the characterisation of focal liver lesions and detection of liver metastases: a systematic review and costeffectiveness analysis. Health Technol Assess. 2013;17(16):1.

6. Mehinovic L, Islamagic E, Husic-Selimovic A, Kurtovic-Kozaric A, Vukobrat-Bijedic Z, Suljevic D. Evaluation of diagnostic efficiency of alpha-fetoprotein in patients with liver cirrhosis and hepatocellular carcinoma: single-center experience. Open 
Karina Dwi Swastika et.al. Accuracy of serum golgi protein 73 and alpha fetoprotein (AFP) to diagnose hepatocellular carcinoma.

Access Maced J Med Sci. 2018;6(9):1668. doi:10.3889/oamjms.2018.344

7. Ali OM, El Amin HA, Sharkawy YL, Mohamed Ali AA, Kholef EFM, Elsewify WAE. Golgi Protein 73 versus AlphaFetoprotein as a New Biomarker in Early Diagnosis of Hepatocellular Carcinoma. Int J Gen Med. 2020 May 18;13:193-200. doi: 10.2147/IJGM.S253622. PMID: 32547160; PMCID: PMC7244238.

8. Soresi M, Magliarisi C, Campagna $\mathrm{P}$, et al. Usefulness of alpha-fetoprotein in the diagnosis of hepatocellular carcinoma. Anticancer Res. 2003;23(2C):1747-1753.

How to cite this article: Swastika KD, Siregar GA, Lindarto D. Accuracy of serum golgi protein 73 and alpha fetoprotein (AFP) to diagnose hepatocellular carcinoma. International Journal of Research and Review. 2021; 8(9): 468-473. DOI: https://doi.org/10. 52403/ijrr.20210959 